\section{La atención a las mujeres diabéticas en el período preconceptivo y la diabetes gestacional ${ }^{1}$}

\section{LA ATENCIÓN A LAS MUJERES DIABÉTICAS EN EL PERÍODO PRECONCEPTIVO}

El objetivo primario de estas directrices consiste en definir los elementos de un programa de atención a las mujeres diabéticas en el período preconceptivo que permita reducir la frecuencia de las malformaciones congénitas. El modelo descrito consta de cuatro elementos principales: 1) la educación de la paciente acerca de las interacciones entre la diabetes, el embarazo y la planificación familiar; 2) la educación sobre el autocontrol de la diabetes; 3) la asistencia médica y las pruebas de laboratorio realizadas por médicos, y 4) el asesoramiento por un profesional de la salud mental cuando sea necesario para reducir el estrés y mejorar el cumplimiento del plan de tratamiento de la diabetes.

\section{Diabetes y malformaciones congénitas}

Las grandes malformaciones congénitas siguen siendo la principal causa de mortalidad y morbilidad grave en lactantes hijos de mujeres con diabetes de tipo 1 o 2 . Varios estudios han establecido una asociación entre las altas tasas de aborto espontáneo y grandes malformaciones y las concentraciones maternas elevadas de glucosa o hemoglobina glucosilada durante el período embriogénico. Por otra parte, varios estudios clínicos han demostrado que el control estricto de la glucemia durante el período preconceptivo y el primer trimestre del embarazo reduce marcadamente la tasa de malformaciones. Lamentablemente, en cerca de dos tercios de las mujeres diabéticas se siguen produciendo embarazos no planeados. Con el fin de reducir la frecuencia de las malformaciones, la atención a las mujeres diabéticas en edad fértil debería incluir: 1) asesoramiento sobre el riesgo de malformaciones asociado a los embarazos no planeados y al mal control metabólico, y 2) el uso eficaz de los métodos anticonceptivos en todo momento, a no ser que la paciente tenga un buen control metabólico y esté tratando de quedar embarazada.

Basado en los documentos "Preconception care of women with diabetes" y "Gestational diabetes mellitus", elaborados por la Asociación Estadounidense de Diabetes (American Diabetes Association) como parte de sus Recomendaciones para la Práctica Clínica del 2001 (Clinical Practice Recommendations 2001), publicadas en Diabetes Care 2001; 24(supl. 1) y disponibles a texto completo, en inglés, en http:// journal.diabetes.org/CareSup1Jan01.htm (acceso el 31 de octubre del 2001).

\section{Diabetes y anticoncepción}

No hay métodos anticonceptivos específicamente contraindicados en mujeres diabéticas, por lo 
que la selección de un método en particular deberá seguir las mismas normas que se aplican a las mujeres no diabéticas, procurando siempre elegir uno de eficacia demostrada.

\section{Programa de atención preconceptiva}

La atención a la diabetes y la educación diabetológica deben comenzar antes de la concepción y lo ideal es que sean realizadas por un equipo multidisciplinario compuesto por un diabetólogo, internista o médico de familia con experiencia en diabetes, un obstetra con experiencia en embarazos de alto riesgo, educadores en diabetes (enfermera, dietista y trabajador social) y otros especialistas que se consideren necesarios.

\section{Objetivos específicos del tratamiento}

El objetivo de la atención médica durante la fase preconceptiva consiste en reducir la hemoglobina glucosilada $\left(\mathrm{HbA}_{1 \mathrm{c}}\right)$ a una concentración que posibilite un desarrollo óptimo durante el período organogénico. Las concentraciones de $\mathrm{HbA}_{1 \mathrm{c}}$ de hasta un $1 \%$ por encima de lo normal no se asocian a tasas de malformaciones y abortos espontáneos superiores a las registradas en embarazadas no diabéticas. No obstante, dichas tasas siguen disminuyendo a medida que se reducen las concentraciones de $\mathrm{HbA}_{1 \mathrm{c}}$. Por consiguiente, el objetivo general debería consistir en obtener la menor concentración posible de $\mathrm{HbA}_{1 c}$ que no conlleve un riesgo inaceptable de hipoglucemia materna. El autocontrol de la enfermedad es fundamental para alcanzar este objetivo y sus requisitos son los mismos que en cualquier programa de autocontrol basado en la insulinoterapia:

- Régimen de comidas adecuado.

- Automonitorización de la glucemia.

- Autoadministración de la insulina y autoajuste de las dosis de insulina.

- Tratamiento de la hipoglucemia.

- Actividad física.

- Técnicas para reducir el estrés y enfrentar la negación.

\section{Consulta inicial}

Antes de planear un embarazo es imprescindible la realización de una anamnesis completa que incluya:

- La duración y tipo (1 o 2) de diabetes.
- Las complicaciones agudas (infecciones, cetoacidosis, hipoglucemia, etc.)

- Las complicaciones crónicas (retinopatía, nefropatía, hipertensión, vasculopatía aterosclerótica y neuropatía periférica y neurovegetativa).

- El tratamiento de la diabetes (régimen de insulina, uso actual o pasado de hipoglucemiantes orales, régimen de automonitorización de la glucemia y sus resultados, tratamiento nutricional y actividad física).

- Medicaciones y trastornos médicos concomitantes, en particular las enfermedades tiroideas en la diabetes de tipo 1.

- Antecedentes menstruales, gestacionales y de uso de anticonceptivos.

- Sistema de apoyo, tanto familiar como en el lugar de trabajo.

En la exploración se debe prestar especial atención a los siguientes aspectos:

- Medición de la tensión arterial y pruebas ortostáticas.

- Fundoscopia con dilatación, realizada por un oftalmólogo o algún otro especialista con conocimientos sobre la afectación ocular de la diabetes.

- Investigación de posibles enfermedades cardiovasculares y, en caso de que se encuentren, realización de pruebas de detección de arteriopatía coronaria antes del embarazo, para comprobar que el aumento de la demanda cardíaca originado por el embarazo será tolerado.

- Exploración neurológica que incluya el sistema neurovegetativo.

Las pruebas de laboratorio deben centrarse en la evaluación del control metabólico y en la detección de complicaciones de la diabetes que puedan afectar al embarazo o verse afectadas por él:

- $\mathrm{HbA}_{1 \mathrm{c}}$.

- Creatinina sérica y excreción urinaria de proteínas totales o albúmina. La proteinuria $>190 \mathrm{mg} /$ $24 \mathrm{~h}$ aumenta el riesgo de trastornos hipertensivos del embarazo, y la proteinuria $>400 \mathrm{mg} /$ $24 \mathrm{~h}$, el riesgo de retraso del crecimiento intrauterino. En estos casos no están indicados tratamientos específicos, pero las pacientes deben conocer los riesgos. Durante el embarazo no se deben tomar inhibidores de la enzima de conversión de la angiotensina (IECA).

- TSH sérica o tiroxina libre en mujeres con diabetes de tipo 1, debido a la coexistencia de hipotiroidismo o hipertiroidismo en el 5 a 10\% de los casos.

- Otras pruebas indicadas por los resultados de la anamnesis o la exploración. 
El plan terapéutico inicial debería incluir los siguientes componentes:

- Asesoramiento sobre el riesgo y la prevención de las malformaciones congénitas, las complicaciones fetales y neonatales de la diabetes materna, los efectos del embarazo sobre las complicaciones de la diabetes materna, el riesgo de complicaciones obstétricas cuya frecuencia está aumentada en las embarazadas diabéticas (en particular los trastornos hipertensivos), la necesidad de anticoncepción eficaz hasta que la glucemia esté bien controlada, y la relación costos-beneficios de la atención preconceptiva y la prevención de las malformaciones.

- Selección del tratamiento hipoglucemiante, que debe consistir en insulinoterapia tanto en la diabetes de tipo 1 como en la de tipo 2, dado que la información existente no permite asegurar la inocuidad de los hipoglucemiantes orales en las fases iniciales del embarazo.

- Establecimiento de un plan para alcanzar una glucemia de bajo riesgo. Para lograr este objetivo se recomiendan dos pasos. En primer lugar, fijar los objetivos de la automonitorización de la glucemia: antes de las comidas, 70 a $100 \mathrm{mg} / \mathrm{dL}$ (3,9 a $5,6 \mathrm{mmol} / \mathrm{L}$ ) en sangre capilar total u 80 a 110 $\mathrm{mg} / \mathrm{dL}(4,4$ a $6,1 \mathrm{mmol} / \mathrm{L})$ en plasma capilar; $2 \mathrm{~h}$ después de las comidas, $<140 \mathrm{mg} / \mathrm{dL}(<7,8$ $\mathrm{mmol} / \mathrm{L}$ ) en sangre capilar total o $<155 \mathrm{mg} / \mathrm{dL}$ $(<8,6 \mathrm{mmol} / \mathrm{L})$ en plasma capilar. Inicialmente se recomienda centrarse en la monitorización de la glucemia preprandial. En segundo lugar, poner en práctica el plan terapéutico y monitorizar la concentración de $\mathrm{HbA}_{1 c^{\prime}}$ principal instrumento para evaluar el riesgo de malformaciones, a intervalos de 1 a 2 meses, hasta que se estabilice. Si no se alcanza la concentración de bajo riesgo deseada $(<1 \%$ por encima del límite superior de la normalidad) habrá que considerar la posibilidad de modificar el tratamiento y añadir la monitorización de la glucemia posprandial. Estos objetivos se pueden alcanzar en régimen ambulatorio.

\section{Atención continua}

Tras la consulta inicial, las pacientes deben ser examinadas cada 1 a 2 meses. También se recomiendan frecuentes contactos telefónicos para ajustar las dosis de insulina y otros aspectos del tratamiento. Una vez que la paciente ha alcanzado concentraciones estables de $\mathrm{HbA}_{1 c^{\prime}}$, se le asesorará sobre el riesgo de malformaciones y abortos espontáneos asociado a dichas concentraciones. Si el riesgo es aceptable y el estado de las complicaciones maternas de la diabetes y de las enfermedades coexistentes lo permite, se puede suspender entonces la anticoncepción. Si el embarazo no tiene lugar en el año siguiente, la paciente debería ser sometida a estudios de fertilidad.

\section{Consideraciones especiales}

El Ensayo Clínico sobre el Control y las Complicaciones de la Diabetes (Diabetes Control and Complications Trial) ha demostrado que los intentos de normalizar el control de la glucemia en pacientes con diabetes de tipo 1 aumenta el riesgo de hipoglucemia grave y ello requiere la inclusión de los familiares del paciente en los programas de educación y control. Los contactos frecuentes con el paciente para reajustar el programa terapéutico forman parte integral de la prevención de la hipoglucemia grave.

La progresión de la retinopatía diabética puede acelerarse durante el embarazo. El riesgo puede reducirse mediante la consecución gradual de un buen control metabólico antes de la concepción y la fotocoagulación preconceptiva con láser en mujeres en las que esté indicada. Esto requiere un examen oftalmológico antes de la concepción. Además, los exámenes oftalmológicos deben seguir a lo largo de la gestación.

La hipertensión es frecuente en la diabetes, bien como complicación o bien como enfermedad concomitante. Además, la hipertensión inducida por el embarazo es otro problema potencial de las diabéticas, sobre todo cuando la proteinuria antes de la concepción o al principio de la gestación es $>190 \mathrm{mg} / 24 \mathrm{~h}$. Se recomienda una cuidadosa monitorización y control de la hipertensión en el período preconceptivo. En mujeres que estén contemplando la posibilidad de quedar embarazadas se deben evitar los IECA, los $\beta$-bloqueantes y los diuréticos.

Antes de la concepción se debe efectuar una evaluación de la función renal mediante la determinación de la creatinina sérica y de alguna medida de la excreción urinaria de proteínas. Estas pruebas se deben repetir periódicamente a lo largo del embarazo, debido a las potenciales repercusiones del embarazo sobre la proteinuria y a las repercusiones de la insuficiencia renal sobre el crecimiento y el desarrollo fetales. A las mujeres con insuficiencia renal incipiente (creatinina sérica $>3 \mathrm{mg} / \mathrm{dL}$ o aclaramiento de creatinina $<50 \mathrm{~mL} / \mathrm{min}$ ) se les debe informar de que el embarazo puede producir un empeoramiento permanente de la función renal en más de un $40 \%$ de los casos. En pacientes con nefropatías menos graves se puede producir un em- 
peoramiento transitorio de la función renal que no contraindica el embarazo.

La neuropatía neurovegetativa puede complicar el tratamiento de la diabetes durante el embarazo. Estas complicaciones deben ser identificadas y tratadas antes de la concepción. El embarazo puede exacerbar la neuropatía periférica y, en particular, los síndromes compartimentales, como el síndrome del túnel del carpo.

La posible existencia de arteriopatía coronaria debe ser investigada siguiendo las directrices de la Asociación Estadounidense de Diabetes (ADA: American Diabetes Association) para el diagnóstico de la cardiopatía coronaria. Se han conseguido llevar a buen término embarazos ocurridos en mujeres diabéticas sometidas a revascularización coronaria. Se debe comprobar que la tolerancia al ejercicio es normal, para aumentar al máximo la probabilidad de que la paciente tolere el aumento de la demanda cardiovascular que supone el embarazo.

El embarazo debe ser confirmado cuanto antes mediante una prueba de laboratorio ( $\beta$-GCh en sangre $\mathrm{u}$ orina). Tras la concepción, la paciente debe ser examinada nuevamente por el equipo sanitario, con el fin de reforzar los objetivos y métodos de tratamiento, que se deben mantener estables a lo largo del primer trimestre.

\section{DIABETES GESTACIONAL}

\section{Definición, detección y diagnóstico}

La diabetes gestacional (DG) se define como cualquier grado de intolerancia a la glucosa que haya comenzado o se haya reconocido por primera vez durante el embarazo, independientemente de que persista o no tras la gestación. Los embarazos complicados con DG representan cerca de un 7\% de todos los embarazos, con oscilaciones entre el 1 y el $14 \%$, dependiendo de la población estudiada y del método de diagnóstico empleado.

En la primera consulta prenatal se debe realizar una valoración del riesgo de DG. Las mujeres cuyas características clínicas indiquen un alto riesgo de DG (obesidad, antecedentes personales de DG, glucosuria o importantes antecedentes familiares de diabetes) deben ser sometidas a determinaciones de la glucemia tan pronto como sea posible. Si no se diagnostica DG en las pruebas iniciales, estas se deben repetir entre las 24 y las 28 semanas de gestación. En mujeres con riesgo intermedio, dichas pruebas se deben realizar por primera vez entre las 24 y las 28 semanas de gestación. Las mujeres con bajo riesgo no requieren este tipo de pruebas, pero solo se pueden considerar como tal aquellas que cumplan todos los requisitos siguientes:

- Edad $<25$ años.

- Peso normal antes del embarazo.

- Pertenencia a un grupo étnico con baja prevalencia de DG.

- Ausencia de antecedentes conocidos de diabetes en los familiares en primer grado.

- Ausencia de antecedentes de tolerancia anormal a la glucosa.

- Ausencia de antecedentes de malos desenlaces obstétricos.

El diagnóstico de diabetes se puede establecer cuando la glucemia plasmática en ayunas es $>126 \mathrm{mg} / \mathrm{dL}$ (> 7,0 mmol/L) o cuando una determinación casual de la glucemia es $>200 \mathrm{mg} / \mathrm{dL}$ (> 11,1 mmol/L), siempre que los resultados se confirmen al día siguiente. En tal caso no es necesario realizar una prueba de sobrecarga de glucosa, pero en ausencia de tal grado de hiperglucemia, el estudio de la DG en mujeres con características de alto riesgo o de riesgo intermedio debe seguir una de las dos estrategias siguientes. La primera, en un solo paso, consiste en la realización de una prueba de tolerancia a la glucosa oral no precedida de una determinación de la glucemia y puede tener una buena relación costo-efectividad en pacientes o poblaciones de alto riesgo. La segunda, en dos pasos, consiste en la realización de una determinación de la glucemia $1 \mathrm{~h}$ después de una sobrecarga oral de $50 \mathrm{~g}$ de glucosa, seguida de una prueba diagnóstica de tolerancia a la glucosa oral en los casos con valores superiores a los normales; cuando se consideran anormales los valores $>140 \mathrm{mg} / \mathrm{dL}(>7,8$ $\mathrm{mmol} / \mathrm{L}$ ), esta estrategia permite identificar a cerca de un $80 \%$ de las mujeres con DG, y la cifra aumenta al $90 \%$ cuando se consideran anormales los valores $>130 \mathrm{mg} / \mathrm{dL}(7,2 \mathrm{mmol} / \mathrm{L})$.

\section{Consideraciones obstétricas y perinatales}

La presencia de hiperglucemia en ayunas (> $105 \mathrm{mg} / \mathrm{dL} \mathrm{o}>5,8 \mathrm{mmol} / \mathrm{L}$ ) puede asociarse a un aumento del riesgo de retraso del crecimiento intrauterino durante las últimas 4 a 8 semanas de gestación. La DG no complicada con menores hiperglucemias en ayunas no se ha asociado a un aumento de la mortalidad perinatal, pero, cualquiera que sea su gravedad, la DG aumenta el riesgo de macrosomía fetal. La DG también puede verse complicada con hipoglucemia, ictericia, policitemia o hipocalcemia neonatales, y se asocia a un aumento de la frecuencia de trastornos hipertensivos maternos y de la necesidad de cesárea. 
Las mujeres con DG corren mayor riesgo de sufrir diabetes, generalmente de tipo 2, tras el embarazo. La obesidad y otros factores promotores de la resistencia a la insulina aumentan el riesgo de diabetes de tipo 2, y los marcadores de autoinmunidad dirigida frente a las células de los islotes aumentan el riesgo de diabetes de tipo 1. Los hijos de mujeres con DG corren mayor riesgo de obesidad, intolerancia a la glucosa y diabetes en la adolescencia tardía y al principio de la edad adulta.

\section{Estrategias terapéuticas durante el embarazo}

La vigilancia metabólica materna debe estar dirigida a la detección de la hiperglucemia que sea suficientemente grave como para aumentar el riesgo fetal. La automonitorización diaria de la glucemia parece ser superior a la monitorización intermitente en la consulta. En mujeres tratadas con insulina hay algunas pruebas de que la monitorización posprandial puede ser superior a la preprandial. La monitorización de la glucosuria no es útil en la DG. La vigilancia materna debe incluir también la tensión arterial y la proteinuria para detectar trastornos hipertensivos. También está indicada una mayor vigilancia en los embarazos con riesgo de muerte fetal, particularmente cuando la glucemia en ayunas es $>105 \mathrm{mg} / \mathrm{dL}$ ( $>5,8 \mathrm{mmol} / \mathrm{L}$ ) o el embarazo sigue más allá del término. Sobre todo al principio del primer trimestre, el estudio ecográfico en busca de un posible crecimiento fetal asimétrico puede ayudar a identificar los fetos que se pueden beneficiar de la insulinoterapia materna.

Todas las mujeres con DG deben recibir asesoramiento nutricional, a ser posible por parte de un dietista. El tratamiento nutricional debe incluir el aporte de las calorías y nutrientes necesarios para cubrir los requisitos del embarazo y debería ser acorde con el objetivo fijado para la glucemia.

En mujeres obesas (índice de masa corporal > 30), una restricción calórica del 30 al 33\% (aproximadamente $25 \mathrm{kcal} / \mathrm{kg} /$ día) reduce la hiperglucemia y los triglicéridos plasmáticos sin aumentar la cetonuria. La reducción de los hidratos de carbono al 35 al $40 \%$ de las calorías reduce la glucemia materna y mejora los desenlaces maternos y fetales.

La insulinoterapia es el tratamiento farmacológico que, añadido al tratamiento nutricional, más reduce la morbilidad fetal. La selección de los embarazos que requieren insulinoterapia puede basarse en determinaciones de la glucemia materna, acompañadas o no del estudio de las características del crecimiento fetal. Cuando se utiliza la glucemia materna, la insulinoterapia está indicada una vez que el tratamiento nutricional no consiga mantener la glucemia en las siguientes cifras:
- Glucemia en ayunas $\leq 95 \mathrm{mg} / \mathrm{dL}(\leq 5,3 \mathrm{mmol} / \mathrm{L})$ en sangre total o $<105 \mathrm{mg} / \mathrm{dL}(<5,8 \mathrm{mmol} / \mathrm{L})$ en plasma.

- Glucemia $1 \mathrm{~h}$ después de la comida $\leq 140 \mathrm{mg} / \mathrm{dL}$ $(\leq 7,8 \mathrm{mmol} / \mathrm{L})$ en sangre total o $\leq 155 \mathrm{mg} / \mathrm{dL}$ $(\leq 8,6 \mathrm{mmol} / \mathrm{L})$ en plasma.

- Glucemia $2 \mathrm{~h}$ después de la comida $\leq 120 \mathrm{mg} / \mathrm{dL}$ $(\leq 6,7 \mathrm{mmol} / \mathrm{L})$ en sangre total o $\leq 130 \mathrm{mg} / \mathrm{dL}$ $(\leq 7,2 \mathrm{mmol} / \mathrm{L})$ en plasma.

La medición del perímetro abdominal fetal al principio del tercer trimestre permite identificar una gran proporción de lactantes sin gran riesgo de macrosomía en ausencia de insulinoterapia materna.

En la insulinoterapia se debe utilizar insulina humana, cuya pauta de administración se guiará por los resultados de la automonitorización de la glucemia. Los hipoglucemiantes orales no son recomendables durante el embarazo porque no hay datos suficientes sobre su seguridad.

El ejercicio físico moderado reduce la glucemia materna en mujeres con DG. Todavía no se conocen sus repercusiones sobre las complicaciones neonatales, pero sus efectos beneficiosos de reducción de la glucemia llevan a recomendar la realización de un programa de ejercicio físico moderado en todas las embarazadas que no tengan contraindicaciones médicas u obstétricas para ello.

En sí misma, la DG no constituye una indicación de cesárea ni de parto antes de las 38 semanas de gestación. La prolongación de la gestación más allá de las 38 semanas incrementa el riesgo de macrosomía fetal sin reducir la tasa de cesáreas, por lo que se recomienda el parto a las 38 semanas, a no ser que existan contraindicaciones obstétricas.

En mujeres con DG se debe alentar la lactancia materna, como en cualquier otro caso.

\section{Consideraciones terapéuticas a largo plazo}

Seis semanas después del parto se debe investigar el estado de la glucemia materna. Si es normal, las determinaciones se deben repetir al menos cada 3 años. En mujeres con deterioro de la glucemia en ayunas o deterioro de la tolerancia a la glucosa tras el parto, estas pruebas se deben repetir una vez al año. Estas mujeres deben ser sometidas a tratamiento nutricional y realizar un programa individualizado de ejercicios físicos, debido a que corren un riesgo muy alto de presentar diabetes. Todas las pacientes con antecedentes de DG deben recibir información acerca de las modificaciones del estilo de vida que reducen la resistencia a la insulina, tales como el mantenimiento de un peso corporal normal a través del tratamiento nutricional y del ejercicio 
físico. En la medida de lo posible, se deben evitar las medicaciones que empeoran la resistencia a la insulina, tales como los glucocorticoesteroides. Los hijos de mujeres con DG deben ser vigilados para detectar la aparición de obesidad o alteraciones de la tolerancia a la glucosa.

\section{SYNOPSIS}

\section{Preconception care for women with diabetes and gestational diabetes}

This piece summarizes two aspects of the "Clinical Practice Recommendations 2001" developed by the American Diabe- tes Association: 1) caring for diabetic women in the preconception period so as to reduce the rates of congenital malformations and of spontaneous abortions, and 2) guidelines for caring for pregnant women with gestational diabetes. For the first aspect, the model of care that is described consists of four primary elements: 1) educating the patient concerning the interactions among diabetes, pregnancy, and contraception; 2) education on diabetes self-control; 3) the medical care provided and the laboratory tests performed by medical personnel; and 4) assistance from a mental health professional when needed to reduce stress and improve compliance with a diabetes treatment plan. For the second aspect there is an analysis of: 1) guidelines for diagnosing gestational diabetes, 2) a set of obstetric and perinatal considerations, and 3) therapeutic strategies to apply during the pregnancy and on a longer-term basis.

\section{Congresos sobre Diabetes}

\section{Diabetes y obesidad}

Fechas: del 7 al 10 de marzo de 2002

Organizador: Organización Panamericana de la Salud

Lugar: Jamaica Grande Resort, Ocho Rios, Jamaica

Información adicional: http://wwwudop.uwimona.edu.jm

Contacto: Srta. Thornia Smith. Tel: 1 (876) 977 1749. Fax: 1 (876) 977 5233. Correo

electrónico: udop@uwimona.edu.jm

62 ${ }^{\text {a }}$ Sesión Científica Anual de la Asociación Estadounidense de Diabetes (ADA)

Fechas: del 14 al 18 de junio de 2002

Organizador: American Diabetes Association (ADA)

Lugar: Moscone Convention Center, San Francisco, California, EE.UU.

Información adicional: http://www.diabetes.org/am02

Contacto: Tel: +1 (800) 232 3472. Fax: +1 (703) 549 1715. Correo electrónico:

meetings@diabetes.org

Congreso Anual de la Asociación Estadounidense de Educadores en Diabetes (AADE)

Fechas: del 7 al 12 de agosto de 2002

Organizador: American Association of Diabetes Educators (AADE)

Lugar: Pennsylvania Convention Center, Philadelphia, EE. UU.

Información adicional: http://www.aadenet.org/annual_frame.html

Contacto: Tel: +1 (800) 338 3633. Fax: +1 (312) 4242427.

$38^{\circ}$ Congreso Anual de la Asociación Europea para el Estudio de la Diabetes (EASD)

Fechas: 1 al 5 de septiembre de 2002

Organizador: European Association for the Study of Diabetes (EASD)

Lugar: Budapest, Hungría

Información adicional: http://www.easd.org/38th/Firstinfo.html

Contacto: Fax: +36 1266 1585. Correo electrónico: benyhe@blavo.hu 